\title{
5. Postgraduate Training and Certification of Physiatrists in Japan
}

Department of Physical Medicine and Rehabilitation, Tohoku University Graduate School of Medicine 出江 紳一

\section{Demographics of Japan}

The population of Japan is 128 million. Among them, the aged population, which is defined as 65 years and over, now stands at more than 19 percent. We have 206 physicians per 100,000 habitants. We are well supplied with physicians in Japan.

\section{Japanese Association of Rehabilitation Medicine (JARM)}

We started the certification system of rehabilitation medicine in 1980. In 1987, the JARM started another certification system following a national global system of certifying specialties in Japan. These two groups of specialists are now called Board-Certified Members of Physiatrists and Board-Certified Members of Rehabilitation Medicine. The present number of members of the JARM is 9449. Among them, 4689 physicians have obtained status as Board-Certified Members of Rehabilitation Medicine, and about 792 are Board-Certified Members of Physiatrists.

\section{Postgraduate training of rehabilitation medicine}

Residency training programs at certified institutes and education courses sponsored by JARM follow the Guidelines for Education of Rehabilitation Medicine, including diagnosis and treatment of dysmobilities, interdisciplinary interventions, and legislative issues. Training in the 1st and 2nd postgraduate years (PGY) includes reading authorized textbooks, and practice of emergency medicine, internal medicine, orthopedics, urological examinations, and so on. In the 3rd and 4th PGYs, we practice rehabilitation for specific disorders, such as stroke, amputation, cerebral palsy, dysphagia, and spasticity. Electrodiagnosis is an essential skill for physiatrists. In the 5th and 6th PGYs, research work as well as the management of interdisciplinary intervention are encouraged. And then, many physiatrists apply for international fellowships. Lectures and workshops are essential for keeping up with current topics as well as renewing certification. The number of courses sponsored by JARM was 36 during the period from April, 1999 to March, 2000. The total number of participants was 3526 , meaning about 100 participants per course. In addition, we have workshops for education on specific clinical categories, such as pediatric rehabilitation, electrodiagnosis, management of neurogenic bladder, and prosthetics and orthotics.

\section{Certification system}

Each of the two board examinations is undertaken every year. The number of applicants for Board-Certified Members of Rehabilitation Medicine has increased rapidly, and the average rate of success was 91\%. As for Certified Members of Physiatrists, about 30-40 new members have been certified each year. The Certified Institution of Rehabilitation Medicine is defined as a hospital where Certified Physiatrists are working. The number of the certified institutions has increased about to 20 new institutions per year. Now we have 358 certified institutions in Japan. Among them, 20 are institutions for pediatric rehabilitation. 


\section{Web-based database}

English abstracts of the articles published in the official journal of JARM are available at http://www.soc.nii.ac.jp/jarm/english/abstract/abstraFm.html. The JARM site also provides a web-based database of evaluation systems used in research articles of rehabilitation medicine made by the Committee of Evaluation and Terminology of JARM (http:// wwwsoc.nii.ac.jp/jarm/hyouka-db1.htm).

\section{Continuing medical education}

Board-Certified Members of Rehabilitation Medicine need to renew certification every 10 years. 2002 was the first year of recertification since the beginning of the certification system. Comparing before and after the recertification, the number of the JARM members and Certified Members of Physiatrists increased, although that of Certified Members of Rehabilitation Medicine decreased. Both chances of education and commitments to the specialty are necessary for continuing medical education. While educational resources of rehabilitation medicine in Japan have increased in quality and quantity, and certification system for physiatrists has been established, collaboration of Board-Certified Physiatrists, such as assembling database, shall be essential for developing educational resources. 